Published in:

Studies in History and Philosophy of Science Part C: Studies in History and Philosophy of Biological and Biomedical Sciences

Volume 41, Issue 4, December 2010, Pages 325-332

http://dx.doi.org/10.1016/j.shpsc.2010.10.003,

NB - this is an author's final version and may differ slightly from the published version.

\title{
Are culture-bound syndromes as real as universally-occurring disorders?
}

\section{Rachel Cooper}

Philosophy, Lancaster University, Lancaster, UK

\begin{abstract}
:
This paper asks what it means to say that a disorder is a "real" disorder and then considers whether culture-bound syndromes are real disorders. Following J.L. Austin I note that when we ask whether some supposed culture-bound syndrome is a real disorder we should start by specifying what possible alternatives we have in mind. We might be asking whether the reported behaviours genuinely occur, i.e. whether the culture-bound syndrome is a genuine phenomenon as opposed to a myth. We might be wondering whether the condition should rightly be considered a disorder, as opposed to some sort of non-disorder condition (e.g. a non-disorder form of deviance, or a potentially valuable condition). We might want to know whether the culture-bound syndrome is really a distinct disorder, in the sense that scientific classification systems should include it as a separate category, or whether it is just a variant of a universally-occurring disorder. I argue that some specific difficulties can arise with determining whether a culture-bound syndrome is a real disorder in each of these three senses. However, the frequent assumption that real disorders will necessarily occur universally, and that those that occur only in certain environments are suspicious is not generally justified.
\end{abstract}

Keywords: culture-bound syndromes, reality, disorder, promiscuous realism, Austin 
While some disorders have afflicted people throughout history and in all cultures, others only seem to occur in highly specific social contexts. It is tempting to think that disorders that occur universally are somehow more "real" than those which only occur in particular cultures. Though this claim is seldom explicitly formulated it lurks behind certain popular forms of reasoning. Consider the debates that occur when the reality of some contested condition is under discussion. Those who claim that the condition is a genuine disorder often seek to support their claim by showing that the condition can be seen to have occurred throughout history or in all places. For example, in his paper “Samuel Pepys and Post-Traumatic Stress Disorder” (1983), published in the British Journal of Psychiatry, R.J. Daly defends the validity of Post Traumatic Stress Disorder by arguing that Pepys' diary entry shows that his contemporaries suffered from PTSD after witnessing the Great Fire of London. Similarly, the proponents of Multiple Personality Disorder have dredged through history looking for cases of multiples (Goff and Simms 1993).

The reverse reasoning also occurs and those who are convinced that some disorder is real frequently assume that this implies that it will be found amongst all human populations. Thus, therapists who are convinced of the reality of PTSD have rushed to offer therapy when disasters afflict nonWestern cultures on the assumption that in so far as PTSD is a genuine disorder it will be found everywhere (Watters 2010). When faced with populations that have faced disaster and yet do not manifest anticipated symptoms these therapists have concluded that cultural norms have inhibited the expression of distress that must really be there.

In this paper I seek to unpack and assess the notion that real disorders must occur universally, and that those that occur only at certain times and in certain places should be viewed with suspicion. I start by considering what it might mean for something to be a "real disorder". I then go on to consider whether it is true that culture-bound syndromes are less real than universally-occurring disorders. The possible links between reality and universality that I will unpack and assess have generally been left tacit, but are nonetheless possible to discern and analyse. I hope to make a convincing case that the tacit beliefs that I uncover have played a key role in reasoning about disorders, and that subjecting them to philosophical analysis is useful, as making such tacit beliefs explicit enables us to clarify them, assess them, and where appropriate reject them.

\section{What counts as a "real disorder"?}


Debates as to whether some particular condition is a "real disorder" are commonplace amongst both healthcare professionals and lay people. Even when talk of "real disorders” is avoided, cognate concepts such as "valid disorders" or "genuine disorders" may be invoked instead. However, despite the centrality of the notion, philosophers generally shy away from speaking of the "real". J.L. Austin's Sense and Sensibilia (1962) provides an exception and provides the classic source for thinking about what it means to describe something as "real". Austin reminds us that asking whether something is real only makes sense when we have some specific alternative in mind. As he puts it:

.... definite sense attaches to the assertion that something is real, a real such-and-such, only in the light of a specific way in which it might be, or might have been, not real..(p.70)

Thus we can ask “Is this real cream?” where the alternative is that it is made from vegetable fat rather than milk. Or, “Is this a real Monet” meaning “Did Monet actually paint this picture?”. Or, “Is that a real ghost?" where the alternatives would be that it's someone wearing a sheet, or I've mistaken a shadow, or something of that sort.

Following Austin’s lead, we can flesh out the question “Are culture-bound syndromes real disorders?” by specifying what alternatives we might have in mind. There are a number of possibilities:

- Do the behaviours that are said to characterise a culture-bound syndrome really occur? I.e. is the culture-bound syndrome a genuine phenomenon as opposed to a myth?

- Is the culture-bound syndrome really a disorder as opposed to a non-disorder state (e.g. a non-disorder form of deviance, or a potentially valuable condition)?

- Is the culture-bound syndrome really a distinct disorder, in the sense that scientific classification systems should include it as a separate category, or is it just a variant of a universally-occurring disorder?

The remainder of the paper considers each possibility in turn. 


\section{Genuine phenomenon or myth?}

The most straightforward way in which a culture-bound syndrome may fail to be a real disorder is if the behaviours that are said to characterise it do not actually occur. By definition culture-bound syndromes are only found in certain cultures. This means that those who would observe cases must travel far and wide, and will frequently have to depend on interpreters. Even then, observing cases first-hand may prove impossible and as a consequence those who describe culture-bound syndromes may resort to relying on reports that are second or even third hand. To make things still worse, many culture-bound syndromes are distinctly "exotic" in character. Stories of penis-shrinking anxieties, of sudden homicidal rages, and of cannibalism, can be expected to be told and retold, and embellished with each retelling. In such a context, distinguishing reliable reports from travellers' tales becomes difficult.

"Windigo Psychosis" may be an example where tales and fact have become confused. Windigo Psychosis has been included in many lists of culture-bound syndromes and is said to be a psychotic state characterised by an obsessional craving for human flesh that occurs amongst the Northern Algonkian peoples. However in his 1982 paper, Lou Marano analyses the case reports that are found in the literature and finds that none provide first-hand accounts of psychotic cannibalism. Though the Northern Algonkian peoples tell stories of Windigo as part of their folklore, and have frequently faced starvation, and thus fear cannibalism, there is little evidence of psychotic cannibalism, as opposed to famine-induced cannibalism, occurring. Marano concludes that "the windigo phenomenon is more of an example of mass suggestibility amongst anthropologists than among Northern Algonkians” (p388).

The fact that culturally-specific phenomena are generally harder to observe than universallyoccurring phenomena gives us the first reason why we might doubt the reality of some culturebound syndromes. The simplest way in which a putative culture-bound syndrome may fail to be a real disorder is if the behaviours that are said to characterise it don't actually occur. This sort of case is included here for completeness, but is discussed only briefly because there is little of philosophical interest to say about such cases. Such fake disorders can be guarded against by common-sense means - here the diligent checking of sources provides the remedy. 


\section{A disorder or a non-disorder state?}

Let us suppose that the behaviours that are said to characterise a culture-bound syndrome genuinely occur. Still the condition may fail to be a real disorder. Real disorders can be confused with various look-a-likes. At the boundaries of disorder lie the following types of non-disorder states: 1. behaviours that may be undesirable but that are under voluntary control - arguably, excessive drinking, shoplifting, and rioting provide examples - these are forms of non-disorder deviance; 2. unpleasant but normal biological and psychological states, such as menstruation and justified sadness; 3. conditions that are unusual but valuable, such as being an especially fast runner, or not having body odour - these are not disorders but are simply manifestations of human diversity.

Difficulties with determining whether a condition is a disorder or another type of condition can arise with both universally-occurring disorders and culture-bound syndromes. As an example of controversial and yet universally-occurring condition consider alcoholism. Alcoholism occurs amongst all cultures with access to alcohol, but whether it should be considered a disorder or a vice is still debatable. However, although difficulties in determining whether a universally-occurring condition should be considered a disorder can occur, specific doubts can arise in the case of culturebound disorders. First, one may have special reason to suspect that in the case of a putative culturebound disorder interested parties have mislabelled a non-disorder state. Second, determining whether a condition is a disorder or non-disorder state can be particularly difficult cross-culturally. I discuss each worry in turn.

\subsection{Mislabelling cases}

In certain circumstances, incentives arise for a non-disorder condition to be mislabelled as a disorder, and where a putative disorder is recognised only in certain cultures we may have reason to be particularly suspicious that this has occurred. Incentives arise for non-disorder states to be labelled disorders because disordered people are treated differently from the healthy. Suffering from a disorder can entitle a person to various benefits, function as an excuse, and also make it the case that a person is seen as an appropriate focus of medical attention. Thus both the "suffering" individuals themselves and those who gain from treating labelled individuals may be motivated to wrongfully claim that some condition is a disorder. Many people are sceptical of the existence of all, or some, mental disorders for such reasons. Most famously, Thomas Szasz claimed that mental illness is a myth, by which he meant that the symptoms of so-called mental illness were either 
veiled communications, or wrongly pathologised “problems in living” (Szasz 1961). Some people are still impressed by Szasz's claims. Still more are sceptical of the existence of at least some particular putative mental disorders. Examples of conditions that remain extremely controversial include Multiple Personality Disorder, Attention Deficit Hyperactivity Disorder, and Social Phobia.

In arguments where one side claims that a disorder is not genuine and the other claims that it is, a common strategy employed by both sides is to examine worldwide or historical prevalence rates. Those who argue that a disorder is made-up, cite statistics that suggest that it is seen only in particular places and periods. Those who argue that it is a genuine disorder, turn to figures that suggest that the rates at which it occurs are roughly uniform worldwide. Such reasoning can be found at all academic levels, from website blogs to top-rated journals. Here are a few examples.

Writing on the web, Tim O'Shea (no date) doubts the existence of Attention Deficit Disorder (ADD). While he accepts that there are some school children who are harder for their teachers to control than others, he suspects that labelling their behaviours as ADD is to mislabel boisterousness. He says:

I had instinctively doubted the existence of ADD from the time when I first began hearing about it. Sounded very suspicious to me. I wondered, why does ADD only exist in the U.S. and not in Scandinavia, not in The Netherlands, not in France, not in Fiji, and not in Japan? A disease that respects geographic borders? Where has it come from all of a sudden, to go from nothing to being a household word in just a few short years?

As another example, in the paper previously cited, R.J.Daly (1992) defends the validity of Post Traumatic Stress Disorder against critics. Critics suspect that those who "suffer from PTSD” are either mislabelling normal but non-pathological reactions to difficult events, or have been schooled to complain of certain symptoms by over-zealous therapists. Daly notes that "Since many of the new writings about post-traumatic stress disorder concern modern experiences and modern responses, which may be culturally determined, it might be reasonable to question the validity and the reliability of diagnosis of this new syndrome” (p.64). His response is to argue that Pepys recorded the symptoms of Post Traumatic Stress Disorder amongst himself and his acquaintances following the Great Fire of London in 1666. This demonstrates the "temporal constancy", and thus, Daly concludes, the validity, of the disorder. 
In debates as to whether a disorder is real, the alternative hypothesis is often that it is some sort of "fake” disorder. Fake disorders are created by mislabelling non-disorder states. At the extremes, the histories of made-up disorders take several forms. Here I will just consider a couple of possibilities for illustrative purposes.

Consider the following history: at stage one, a small number of individuals spontaneously adopt some behaviour which receives attention from the medical community and is a candidate for diagnosis. The condition also brings advantages for those who "suffer" from it, or for those who stand to gain from them, and is publicized by the media. At stage two, other individuals intentionally copy the behaviour in order to reap the same rewards, and an epidemic of a new “disorder” occurs. In Ian Hacking's paper [this volume] cynics saw the epidemic disorder amongst refugee children in Sweden as a made-up disorder of this type. They claimed that children were faking their symptoms in order to be granted refugee status. In such cases, a non-disorder form of deviance is mislabelled as a disorder.

As another example, consider a case where some segment of normal human variation becomes pathologised. Arguably this has occurred in some cases as a result of marketing campaigns by pharmaceutical companies who can thereby create profitable new markets. Christopher Lane (2007) argues that this has occurred in the case of shyness, which in its extreme form has been rebranded as Social Anxiety Disorder. If Lane is right we can expect cases of Social Anxiety Disorder to track marketing campaigns.

When the alternative to a disorder being genuine is that it is a made-up disorder, prevalence rates of the condition as it occurs around the world or in the past can help to determine the issue. If it is the case that the "sufferers" of a condition are merely copying recently publicised behaviours then cases of the "disorder" should not be found prior to the condition coming to notice. Similarly, a "disorder" that has been created by a marketing campaign will not have caused complaints beyond the campaign's reaches.

When a disorder can be seen to occur throughout the world and at all times, this can often rule out various "fakery" hypotheses concerning its origins. However, a caveat must be noted. Data regarding incidence rates needs to be treated with caution - as sometimes the "universal occurrence" of a disorder can be created rather than discovered. This can occur because institutions and ways of doing medicine get exported from one place to others. 
In Pharmaceutical Reason (2005) Andrew Lakoff looks at the ways in which US-style psychiatric diagnoses have come to be exported to Argentina. International biomedical companies had found it hard to conduct research or sell their products in Argentina because the psychiatric conditions at which their products were targeted were simply not being seen. Mental health professionals in Argentina tended to think in psychoanalytic terms and thus failed to diagnose the sorts of conditions - depression, anxiety, and so on - for which drugs are designed. To address this problem a whole way of practising psychiatry had to be exported. The companies sponsored training events and distributed drug samples and key texts. The end result was that Argentine patients came to be diagnosed with the same conditions as those found in the U.S.. Kalman Applbaum (2006) tells a similar tale about the marketing of depression and the SSRIs that treat it in Japan. When the prevalence rates of a disorder in a culture only approach international norms once such priming activities have occurred, interpreting them becomes difficult. Were cases of the disorder always there, but only recently recognised? Or, have the patients' symptoms somehow been shaped by the newly exported medical system? Still, at least in cases where a condition can be found in different cultures without such priming activities occurring, the existence of a disorder in all times and places suggests that it is a genuine, as opposed to made-up, disorder.

Universally-occurring disorders will not be fake disorders. However, the reverse conclusion cannot justifiably be drawn. Disorders that are found only in certain places and times need not be fakes. In some cases, localised disorders have a biological basis. Here kuru is a key example. Kuru occurred amongst the Fore group in Papua New Guinea mainly from the 1920s until the late 1950s (though a few cases still arise) (Collinge et al 2006). It is caused by a prion (like scrapie in sheep) and was transmitted amongst the Fore, because the Fore ate their dead. Disorders that are caused by drugs, poisons, or nutritional deficiencies will also only occur in certain environments. Heroin addiction requires a population that uses heroin, lead poisoning will increase with lead piping, and cretinism tracks iodine-poor diets.

Culture-bound syndromes where no biological basis is known may also still be genuine disorders as opposed to fakes. Indeed in many cases the most plausible hypotheses concerning the origins of a disorder are not that it has deliberately been made-up but that some more subtle effect of cultural patterning is in operation. In Rewriting the Soul (1995), Ian Hacking suggests that when cases of Multiple Personality Disorder (MPD) came to media attention they supplied a template for a way in which distress could be made manifest. Hacking doesn't claim that patients consciously mimicked 
the symptoms of MPD but rather that unconscious suggestion led to patients who had come across the template manifesting similar symptoms when they would not otherwise have done so. Somewhat similarly, in his analysis of an epidemic mental disorder amongst refugee children in Sweden, Hacking (this volume) argues that an Imitation and Internalisation model best explains the phenomenon. According to this model, the children first begin to more or less voluntarily imitate the behaviour of others, but then find that they lose control and come to manifest symptoms involuntarily. In such cases, Hacking suggests, the disorder is "real” in that the distress is real, and the symptoms are, at least eventually, genuinely not under conscious control, but still it is the case that the disorder only exists within a certain social setting.

In this paper I have separated out the questions of whether a condition is really a disorder, as opposed to a non-disorder condition, and the question of whether it is a really a distinct disorder in the sense that scientific classification systems should include it as a separate category, as opposed to being merely a variant of a universally-occurring disorder. Keeping these questions separate can help us see why Hacking is correct to claim that conditions that occur only in very particular environments can be real disorders, but how there might still be reason to find such cases problematic. When a condition occurs only in very particular cultural contexts I suggest we can often fairly consider the condition to be a real disorder as opposed to a non-disorder condition, but that we might still suspect that it is best considered a variant of some more universally-occurring condition rather than being a distinct condition. The disorder that occurred amongst Swedish refugee children is a real disorder rather than a non-disorder condition in that it is undesirable, it is involuntary, and it is not normal. Still, in so far as it only occurs in very specific contexts we know that its form is fragile, and with a slight change in cultural conditions the particular syndrome will dissolve. This might lead us into arguments when it comes to classification. Maybe rather than the refugee children manifesting a distinct disorder they should be seen as suffering from a form of a universally-occurring disorder - maybe, for example, a type of depression. I return to this question in section four.

\subsection{Cross-cultural difficulties}

On many accounts of disorder, a condition can only be a disorder if it is harmful (Flew 1973, Reznek 1987, Wakefield 1992, Cooper 2002). A condition that causes no harm is not a disorder but merely a manifestation of human diversity. Working out whether a condition is harmful can 
sometimes be problematic. Consider, for example, contemporary debates over Deafness, which Deaf activists claim is a valuable condition as opposed to a disability (Cooper 2007). The difficulties inherent in determining whether a condition is a bad thing are magnified in crosscultural contexts, as across cultures both the environment in which a person must live and social judgements of the good life will vary.

Clearly whether or not a condition will be harmful depends on the physical and social environment in which it occurs. To be dyslexic in a literate culture is far worse than being dyslexic in a culture in which no one reads or writes, and so on accounts according to which disorders must be harmful, dyslexia can be considered a disorder in some environments but not in others. Even when cultures agree that a state is somewhat undesirable, assessments of how problematic it is can vary, and this will lead to the threshold between the pathological and the normal being drawn differently. Chinese culture traditionally discourages the expression of strong emotions. Glorisa Canino and Margarita Alegria (2008) suggest that this may lead Chinese parents and clinicians to have a lower tolerance for hyperactive behaviour, and as a result ADHD is diagnosed in children twice as frequently in Hong Kong as in the US. Cases like these occur because in different societies a human needs different attributes in order to fit in.

Cultures also differ in the value that they attach to different experiences. Japanese culture has traditionally seen value in melancholic moods (Watters 2010 Ch. 4). Such states are considered to be linked to praiseworthy traits of diligence, care, and concern for others, and to provide a source of moral meaning and self-understanding. This stance has meant that while Western psychiatrists have been happy to diagnose fairly mild conditions as forms of depression, their Japanese counterparts have seen such states as non-pathological. In a similar vein, Gloria DuraVila and S. Dein (2009) describe the Dark Night of the Soul as experienced by Spanish Catholic nuns from a contemplative order. The Dark Night is a period of spiritual crisis. When going through the Dark Night the nuns typically experience symptoms that are normally linked with pathological depression (feelings of misery and worthlessness, tearfulness, loss of appetite). However, the nuns see the Dark Night as non-pathological, as a normal and valuable part of spiritual growth.

In evaluating states which some cultures consider beneficial and others consider harmful, we face head-on the difficult question of what determines whether a human being has a good life. There are a range of options. As a non-exhaustive list, one might claim that whether a human 
has a good life depends on any of the following:

- How that individual evaluates their life.

- How that individual's peers evaluate their life

- On some objective standard (neo-Aristotelian accounts, such as the capabilities accounts put forward by Amartya Sen (1980) and Martha Nussbaum (2006) are examples of such accounts).

Whichever option one takes difficulties instantly emerge. Claiming that an individual's life is good because they themselves evaluate it as such runs into problems with subjects who are deluded, or simply foolish. Relying on cultural standards is problematic as whole cultures can surely be mistaken about the good life (many cultures have considered homosexuality deeply harmful, or slavery acceptable). If one claims that there are objective standards for the good life, then the challenge is to work out how these standards are determined. The question of how to determine the good life for an individual is extremely difficult, and certainly won't be resolved here. Interested readers might consult Griffin (1986) Well-being which very usefully lays out the various possibilities and the difficulties that they face. Here I just note that disagreements over the nature of the good life will be particularly problematic across cultures, and can lead to disagreements as to whether a particular condition should be considered a disorder or not. Such difficulties mean that determining whether a putative culture-bound syndrome should be considered a disorder or is instead a potentially valuable state and thus merely a manifestation of human diversity can be especially problematic.

Determining whether a condition is under voluntary control can also be especially difficult in cross-cultural settings. Only involuntary conditions are disorders; voluntary but undesirable behaviours are non-disorder forms of deviance. Working out whether a state is voluntary depends on an assessment of subtle psychological and social cues. Getting such assessments right is difficult enough when considering people from one’s own social group, and when considering the behaviours of people from other cultures the difficulties are greatly magnified. As an example, consider Latah which is a condition found mainly amongst Malaysian women. Latahs are said to be hypersensitive to startling events, which allegedly cause them to enter trance-like states in which they may automatically imitate others, obey commands, and use foul language. Bartholomew (1999) suggests that Latahs can actually control their behaviour and that thus Latah should not be considered a disorder.

Some conditions are not disorders because they are simply normal, though unpleasant, human 
states. Menstruation and teething are examples. Again determining whether a condition is a disorder or a normal state can be particularly difficult across cultures. People who live in different environments differ biologically, for example depending on the diets of their ancestors current populations differ in their tolerance to alcohol and lactose (Omenn 2010). Figuring out whether a condition is pathological for a given individual can thus become problematic. Depending on the population with which they are compared, their condition may or may not be considered normal.

\section{Real and independent_category or variant of other disorders?}

On some influential views of the role of classification in science, scientific kinds must be such that they can potentially be found anywhere. For example, the New Essentialism propounded by Brian Ellis (2002) holds that classification is useful in so far as it picks out natural kinds and that natural kinds have intrinsic essential properties. Such an account sees the basic categories that are recognized by physics and chemistry as providing paradigmatic examples of the types of kind that science should seek to identify. On such an account, kinds that are defined in an essentially historical manner, such as biological species, which on many accounts of the species concept should be defined in terms of evolutionary lineage, have only a secondary status.

It is beyond the scope of this paper to assess the claim that kinds that are defined in an essentially historical manner are somehow of a lesser status than those that can be defined in terms of intrinsic properties. It is sufficient for my purposes to note that sciences such as biology and geology do usefully recognize categories of entity that can only be found in certain locations or in certain periods. In order to avoid debates about the character of "natural kinds", let's call the kinds that are recognised by established sciences - which will include physics, chemistry, biology and geology - “useful scientific kinds”. Here I will ask whether culturebound syndromes might be useful scientific kinds in the same sort of way as are biological species or types of rock. In this comparison the fact that culture-bound syndromes can only occur in certain locations does not in itself rule them out as useful scientific kinds.

Still the question emerges as to whether the various culture-bound syndromes should be recognized as distinct disorders or should be considered to be mere variants of other disorders. I suggest that there will plausibly be no one solution here. Some culture-bound syndromes may 
best be considered variants, others may be completely distinct disorders, and some may turn out to be usefully thought of as being both - in the sense that they form a distinct subtype of a more universally-occurring category of disorder. In any case we should avoid the assumption that the Western variants of disorders are somehow “purer” and "less culture-bound" than those that occur in other cultures. In some cases it may be the Western form of a disorder that is best seen as a mere variant of a more universal category.

A useful picture for thinking about the categories of disorder that one might recognize can be supplied by the philosopher John Dupré’s account of Promiscuous Realism (1981, 1993). Dupré asks us to imagine the individual entities of some domain mapped out on a multidimensional quality space (that is a multidimensional graph where the dimensions correspond to different qualities). In such a space, two entities with identical properties end up at the same point, while two entities with very different properties end up far from each other on the graph. Dupré claims that if we plotted the entities in the world onto a multidimensional quality space we would find numerous clusters corresponding to groups of similar entities.

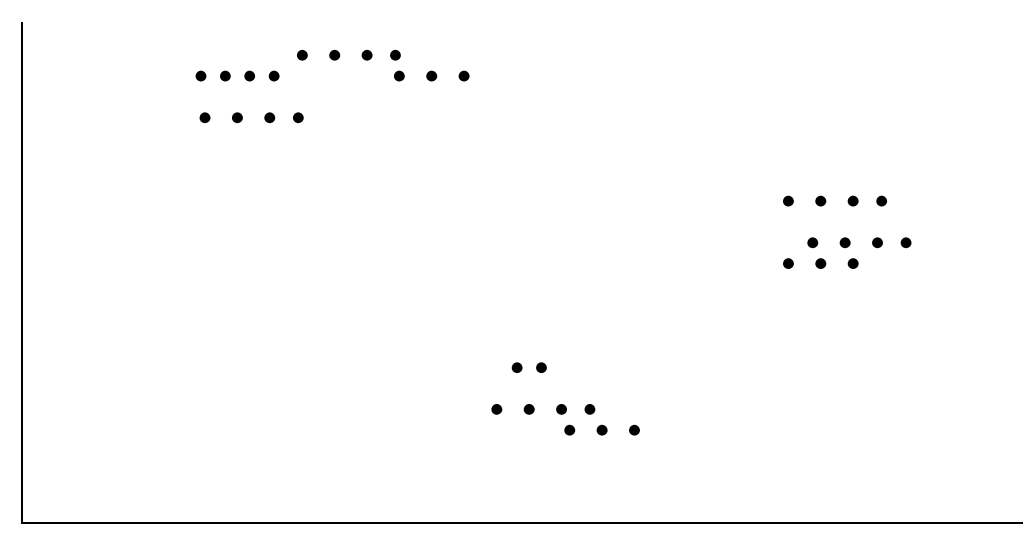

Fig 1: Two dimensions of a multidimensional quality space, showing three clusters of similar entities.

Dupré is interested primarily in biological kinds, but we can also make use of his ideas when thinking about psychopathology. If we were to apply a Dupré-style account to psychopathology, we would expect some clusters to correspond to traditional categories of mental disorder. In addition, at different levels of resolution it will also be possible to make out other clusters. In many cases the clusters will not be discrete, but will be messy and hard to make out. Different clusters can also be generated by restricting our attention to particular dimensions of the map. If we are particularly 
interested in phenomenology, or in treatment-response, we can concentrate on those dimensions. Dupré's account is realist because the clusters in quality space reflect the real structure of nature. It is promiscuous because there will be many different clusters on which we could choose to focus.

On Dupré's picture, we can recognise categories within categories. Furthermore, categories at different levels needn't be strictly hierarchical, they can also be criss-crossing. This means that we don't need to decide to treat a culture-bound syndrome as either a distinct category, or as a mere variant of a universally-occurring condition. Here we can have our cake and eat it, and if it would be useful in a particular case, we can say that a particular disorder is both plausibly a variant of a universally-occurring condition and also that it should be considered as a distinct category in its own right. There is nothing suspicious about a classification system that takes such a line. Biological classifications commonly work in this way - the orang-utans that live in Borneo are a different race from those that live in Sumatra. Sometimes this is important, and scientists can fairly say that the two races are importantly distinct. In other cases, the similarities may be more important than the differences, and in these situations a scientist can fairly conclude that the two races of orang-utan are merely variants of a more universal type.

Returning to psychopathology, a paper by Lewis-Fernández, Guarnaccia et al (2002), examining the phenomenology of ataques de nervios, panic attacks and panic disorder nicely illustrates why it might be useful to recognise multiple and complex categories of disorder. Ataques de nervios is a culture-bound syndrome that occurs principally amongst Latinos from the Caribbean, but also amongst various Latin American groups. An ataque is typically triggered by a stressful event, such as hearing of the death of a loved one, and is characterised by a sense of being out of control, coupled with symptoms such as crying, trembling, and uncontrollable shouting or acts of aggression. Ataques de nervios and panic attacks overlap, in that many of those who meet the criteria for one also meet criteria for the other. This might lead one to want to sometimes group them together, as both being variants of some more general anxiety-type reaction. On the other hand, Lewis-Fernández, Guarnaccia et al found potentially important phenomenological differences between some ataques and panic disorder episodes. In particular, while the symptoms of panic attacks peak in less than ten minutes, those associated with some ataques take longer. The difference in the speed with which symptoms develop is potentially important as certain models of panic posit that "once the attack begins, the neurochemical processes trigger a rapid crescendo of symptoms which peak in less than 10 minutes” (p210). Thus, for certain investigations, keeping ataques de nervios and panic disorder 
apart may be important. To complicate things further, the relationship between "anxiety reaction" and "ataques de nervios" may not be strictly hierarchical as some ataques seem to be characterised more by anger than anxiety (p.216). On a promiscuous realist account researchers can legitimately pick and choose, and in different investigations that can employ different classifications.

While many kinds can usefully be recognised, there may be some that occur so messily, or so fleetingly, that there is no point in recognising them as distinct kinds. Messy and fragile kinds occur in many domains. From botany, consider kinds of bramble (Rubus). Brambles can reproduce either sexually or asexually and they hybridise easily. This means that numerous local varieties emerge. The bushes in one village may differ from all other brambles, and yet a classification system that recognised too many types of bramble would be of little use. Faced with the complexity of their domain, batologists (bramble specialists) have been forced to adopt conventions that determine when a new species can be recognised. To a first approximation, only those biotypes that are spread over a distribution area with a diameter of at least $50 \mathrm{~km}$ can be recognised as distinct species (see Weber 1996 for further details). This rule is clearly merely a convention, but it acts to keep the number of recognised bramble-species in check. In so far as culture-bound syndromes turn out to be messy or fragile kinds there might similarly be reasons for imposing restrictions on the conditions under which scientific classification systems should recognise new distinct disorders.

In some cases we may want to consider cases of a disorder that are phenomenologically unalike to be co-members of a kind. Currently, for example, many psychiatrists are happy to talk of “masked depression”. In particular, claims have been made that the somatic complaints of Chinese people which Chinese practitioners diagnose as neurasthenia, should be considered a form of Western depression (Parker, Gladstone and Tsee Chee 2001). Note that Western depression and Chinese neurasthenia are phenomenologically unalike - the Chinese sufferers do not feel depressed. As Jennifer Radden (2003) notes, grouping phenomenologically distinct conditions together only makes sense if one adopts a causal, as opposed to descriptive, account of the semantics of disease-terms. Only on a causal account, according to which "depression" refers to all disorders that have a certain underlying cause, can one sensibly claim that one disorder might manifest itself very differently in different cultures. On a descriptive account, according to which "depression” refers to disorders characterised by depressive symptoms, the idea that there might be forms of depression that manifest with atypical symptoms makes no 
sense.

For cases of Chinese neurasthenia and Western depression to be co-members of a kind one must adopt a causal account of the semantics of disease-terms. In addition, it needs to be the case that the underlying causes of the phenomenologically distinct states really are alike. Determining whether this is so requires one to utilise theories of psychopathology. Only cases that the theory says have the same sort of cause can be grouped together. In so far as different cultures have different theories regarding the root causes of disease, when they seek to classify by causes, they will group disorders differently. For example, cultures that believe that spirits cause disease will group together cases of disorder that are believed to be caused by similar spirits even if they are phenomenologically distinct.

On a Dupré-style promiscuous realist picture there are many possible legitimate classifications. We can generate different classifications by focusing on different dimensions in the multidimensional property space, or by considering different levels of resolution. However it is not the case that anything goes. Only cases that are genuinely similar in some respect can justifiably be grouped together. Thus when we seek to classify by underlying causes, our causal claims must be roughly correct, at least to the extent that cases that are claimed to have similar causes really do have similar causes. Those classifications that are generated by theories that turn out to be radically wrong must be rejected as illegitimate. In so far as different cultures have radically different theories regarding the causes of disease many cultures will turn out to be completely wrong. Of course, in some cases the theories of non-Western cultures may turn out to be closer to the mark than those of Western medicine, but the diversity of theories implies that many of the categories of disease that are currently recognised across the world should eventually be eliminated on the basis that they group together cases that are not actually alike (for further discussion on the conditions under which a folk category should be eliminated see Blease, this volume).

Legitimate categories of disorder should group together cases that are alike in some respect. Does it matter whether the properties that co-occur are glued together by biology or culture? The idea that real disorders must have a presumed biological basis is popular, and on such accounts in so far as culture-bound syndromes are paradigmatically culturally-shaped they cannot be real disorders. Adopting such a position, in Creating Mental Illness (2002) Allan Horwitz argues that most nonpsychotic mental illnesses are not genuinely distinct conditions 
because "symptoms stem from general underlying vulnerabilities that may assume many different overt forms, depending on the cultural context in which they arise...Cultural processes, not the unfolding of natural disease, structure the overt manifestation of symptoms into recognizable entities" (p.108). Horwitz argues that whether a vulnerable and distressed person manifests the symptoms of depression, or an anxiety disorder, or a phobia, or a somatization disorder, or some other disorder, depends on their cultural context.

Against Horwitz, I suggest that syndromes that are glued together by culture can be as scientifically respectable as those that are glued together by biology. Here we can consider some of the kinds that occur in the natural historical sciences for illumination. Specifically, I want to consider the different sorts of igneous rocks that are recognised by geologists. These rocks are all formed from variations of the same basic stuff - magma. However their characteristics vary depending on the conditions under which they were formed. For example, slow cooling rocks have large crystals and fast cooling rocks have small crystals. The rocks are classified according to their chemical composition and their history (both of which can vary in complex and continuous ways). I suggest that, if Horwitz is right and different varieties of anxiety-depression type disorders are formed into distinct entities by cultural context, then we can think of such disorders as being kinds analogous to the different kinds of rock distinguished by geologists. In both cases a basic amorphous "material” would come to take different forms depending on the history of its formation.

Kinds that only occur in specific historical contexts - such as kinds of rock, and culturally formed type of mental disorder - can usefully be considered scientific kinds, I suggest, because they can support explanations and inductive inferences and feature in law-like generalisations. The individuals that fall into such kinds are "repeatables" in the sense that any two specimens of basalt, or any two cases of anorexia, can be expected to have much in common. Admittedly the members of such kinds are only found in certain environments. If cultural conditions shift, then cases of anorexia may no longer occur. Similarly, rocks created under certain environmental conditions may never form again if the conditions cannot be repeated. Still, though cases may occur for a limited time or in limited places, within those constraints the kinds are as scientifically useful as those that occur universally.

The example of magma and igneous rocks helps show that historically situated kinds can be scientifically useful. However the example has its limitations. In the case of magma and 
igneous rocks a uniform basic stuff comes to manifest different properties depending on history. The relationship between basic biology and culturally shaped disease manifestation may sometimes be like that but more complex interactions also occur. Diet, child-rearing practices, and patterns of work and leisure are all culturally-influenced and have biological effects. As Laurence Kirmayer puts it “The biological effects of diet and lifestyle break down the culture/ nature dichotomy, since culturally determined patterns of behaviour refashion human biology” (2006, p129). Still the key point remains, within a particular human population biology and culture may interact so as to produce cases of a disorder that are recognisably and reliably similar to each other and such disorders can usefully be recognised by psychiatric classification systems.

\section{Conclusions and Implications}

Compared to universally-occurring disorders there are some specific difficulties with determining whether a culture-bound syndrome is a real disorder. Following J.L. Austin I have noted that when we ask whether some supposed culture-bound syndrome is a real disorder it is best to start by specifying what possible alternatives we have in mind. We might be asking whether the reported behaviours genuinely occur. We might be wondering whether the condition should rightly be considered a disorder, as opposed to some sort of non-disorder condition. We might want to know whether the condition should be considered a distinct type of disorder as opposed to being merely a variation of a universally-occurring disorder.

In cases where the alternative is that the reported phenomena do not actually occur, we can note that phenomena that only occur in certain cultures tend to be harder to observe than those that occur universally. This increases the dangers that the behaviours that are said to characterise a supposed disorder have been misreported and do not actually arise. Windigo psychosis is a possible example of a condition that fails to be a real disorder because it is just a travellers' tale.

In cases where the alternative is that the condition is really some non-disorder state as opposed to a disorder, specific difficulties also arise in the case of culture-bound syndromes. Incentives can exist for interested parties to pathologise non-disorder states. Here disorders that can be seen to occur at diverse places and times are on a surer footing because they cannot have been constructed with the intention of achieving objectives that are specific to a local context. In addition, determining whether a condition is a disorder or a potentially valuable state, whether 
it is involuntary, and whether it is simply normal for a given population, can all be particularly difficult cross-culturally.

In considering whether a culture-bound syndrome should be seen as a distinct disorder or simply a variation of a universally-occurring disorder we have seen how Dupré's promiscuous realism offers a model on which one can entertain a multitude of taxonomic possibilities. A culture-bound syndrome can be both a local variant, and also worthy of a distinct category - as different classifications may concentrate on different aspects of psychopathology and be used for different purposes.

Although there are circumstances in which we might be particularly suspicious of disorders that are reported only in certain cultures, some culture-bound syndromes will be real disorders in all three senses considered here. That is, some culture-bound syndromes will be real disorders in that they are genuine phenomena, they really are disorders as opposed to non-disorder conditions, and they can fairly be considered distinct disorders as opposed to merely being variants of universally-occurring disorders. Thus the frequent assumption that real disorders will necessarily occur universally, and that those that occur only in certain environments are suspicious is not generally justified.

We can conclude that some real disorders are culture-bound. This has important implications. To give just one example, the assumption that in so far as a disorder is real it will occur everywhere has motivated philanthropic mental health interventions in developing countries (Watters 2010). Western mental health professionals, who are convinced that disorders such as eating disorders, PTSD, and depression are real, have noticed the absence of services in other cultures and have assumed that this means that suffering populations are being neglected. This has led them to set up Western-style mental health services across the globe. Once one appreciates that real disorders may not occur everywhere, the assumption that such services can usefully be exported from one cultural setting to another is undermined. Of course, sometimes non-Western populations will suffer from the conditions that afflict Westerners and setting up services will be helpful, but such needs should be established rather than assumed. 


\section{Acknowledgements}

A version of this paper was presented at a workshop on Culture-Bound Syndromes, held at Lancaster University in 2009, as part of the AHRC funded research network onThe Concepts of Health, Illness and Disease. I am very grateful for the comments of those present.

\section{References}

American Psychiatric Association (2000). Diagnostic and Statistical Manual of Mental

Disorders (Fourth Edition Text Revision). Washington: American Psychiatric Association.

Applbaum, K. (2006). Educating for global mental health: The adoption of SSRIs in Japan. In A. Petryna, A. Lakoff, \& A. Kleinman (Eds.), Global Pharmaceuticals: Ethics, Markets, Practices (pp.85-110). Durham: Duke University Press.

Austin, J.L. (1962). Sense and Sensibilia. London: Oxford University Press.

Bartholomew, R. (1999). The conspicious absence of a single credible case of Latah-related death or serious injury. Transcultural Psychiatry, 36, 369-376.

Canino, G. and Alegria, M. (2008). Psychiatric diagnosis - is it universal or relative to culture? The Journal of Child Psychology and Psychiatry, 49, 237-250.

Collinge, J., Whitfield, J., McKintosh, E., Beck, J., Mead, S., Thomas, D., Alpers, M. (2006). Kuru in the $21^{\text {st }}$ century - an acquired human prion disease with very long incubation periods. Lancet, 367, 2068-74.

Cooper, R. (2002). Disease. Studies in History and Philosophy of Biological and Biomedical Sciences, 33, 263-282.

Cooper, R. (2007). Can it be a good thing to be Deaf? Journal of Medicine and Philosophy, 32, 563-583.

Daly, R. (1983). Samuel Pepys and Post-Traumatic Stress Disorder. British Journal of 
Psychiatry, 143, 64-68.

Dupré, J. (1981). Natural kinds and biological taxa. The Philosophical Review, XC, 66-90.

Dura-Vila, G. \& Dein, S. (2009). The Dark Night of the Soul: spiritual distress and its psychiatric implications. Mental Health, Religion and Culture, 12, 543-559.

Ellis, B. (2002). The Philosophy of Nature. Chesham: Acumen.

Flew, A. (1973). Crime or Disease? London: Macmillan.

Goff, D. \& Simms, C. (1993). Has Multiple Personality Disorder remained consistent over time? A comparison of past and recent cases. The Journal of Nervous and Mental Disease, 181, 595-600.

Griffin, J. (1986) Well-Being. Oxford: Clarendon Press.

Hacking, I. (1995). Rewriting the Soul. Princeton: Princeton University Press.

Horwitz, A. (2002). Creating Mental Illness. Chicago: University of Chicago Press.

Kirmayer, L. (2006). Beyond the 'New cross-cultural psychiatry': cultural biology, discursive psychology and the ironies of globalization. Transcultural Psychiatry, 43, 126-144.

Lane, C. (2007). Shyness: How normal behaviour became a sickness. New Haven: Yale University Press.

Lewis-Fernández, R., Guarnaccia, P., Martinez, I., Salmán, E., Schmidt, A. \& Liebowitz, M. (2002) Comparative phenomenology of ataques de nervios, panic attacks, and panic disorder. Culture, Medicine and Psychiatry, 26, 199-223.

Marano, L. (1982). Windigo psychosis: The anatomy of an emic-etic confusion. Current Anthropology, 23, 385-412. 
Nussbaum, M. (2006). Frontiers of Justice: Disability, Nationality, Species Membership.

Harvard: Harvard University Press.

Omenn, G. (2010). Evolution and public health. Proceedings of the National Academy of Sciences of the United States, 107 (suppl. 1), 1702-1709.

O'Shea, T. (no date). ADD/ADHD: The "Designer Disease".

http://www.chiro.org/pediatrics/ABSTRACTS/Designer_Disease.shtml (Accessed 14 January 2010)

Parker, G., Gladstone, G. \& Tsee Chee K. (2001). Depression in the planet's largest ethnic group: The Chinese. American Journal of Psychiatry, 158, 857-864.

Radden, J. (2003). Is this Dame Melancholy? Equating today's depression and past melancholia. Reprinted in J. Radden (2009) Moody Minds Distempered: Essays on Melancholy and Depression. (pp.75-93) Oxford: Oxford University Press.

Reznek, L. (1987). The Nature of Disease. London: Routledge and Kegan Paul.

Sen, A. (1980). Equality of what? in S. McMurrin (Ed.), The Tanner Lectures on Human Value (pp. 195-220). Salt Lake City: University of Utah Press.

Wakefield, J. (1992). The concept of mental disorder - On the boundary between biological facts and social value. American Psychologist, 47, 373-388.

Watters, E. (2010) Crazy Like Us: The Globalization of the American Psyche. New York: Free Press.

Weber, H. (1996). Former and modern taxonomic treatment of the apomictic rubus complex. Folia Geobotanica and Phytotaxonomica, 31, 373-380. 
